\title{
SEXUAL SELECTION FOR DOMINANT AND RECESSIVE GENES
}

\author{
P. O'DONALD
}

Department of Zoology, University College of North Wales, Bangor

\section{INTRODUCTION}

Received 9.iv.63

IN Fisher's theory of sexual selection (Fisher, I930) the females that exercise an hereditary mating preference for males favoured in natural selection are themselves selected by the selective advantage of their sons who will possess the advantageous male genotypes and at the same time will carry the mating preference genes. Once established the mating preference itself adds to the selective advantage of the preferred genotypes: as Fisher suggests a " runaway process" develops.

In a paper in Heredity, vol. I 7 ( 1962 ), I described a model of this kind of selection. I assumed that two alleles $\mathrm{A}$ and $\mathrm{B}$ determined the male character and an allele $\alpha$ linked to the X-chromosome determined the female preference. In birds to which the model applies the female is the heterogametic sex: females who are $\mathrm{X}^{a} \mathrm{Y}$ mate only with $\mathrm{AA}$ males, and females who are $\mathrm{X}+\mathrm{Y}$ mate with $\mathrm{AA}, \mathrm{AB}$ and $\mathrm{BB}$ males at random. The recurrence relations $I$ obtained were non-linear and I used the model to give only a rough idea of the rate of selection. It did seem clear, however, that if A were closely linked to $a$ selection would be very fast. If selection were to pick out those genes for male display that were more closely linked to the mating preference genes, a supergene controlling both male display and the female response to it would evolve.

In my original model the $\mathrm{X}^{a} \mathrm{Y}$ females select the allele $\mathrm{A}$ as a recessive. In this paper I extend the model in two ways-sexual selection at an autosomal locus and sexual selection of both dominant and recessive genes.

TABLE

\begin{tabular}{|c|c|c|c|c|c|c|c|c|}
\hline & & & \multicolumn{6}{|c|}{ Genotypes and their frequencies in the females } \\
\hline & & & $\underset{\mathbf{X}^{a \mathbf{Y}}}{\mathrm{AA}}$ & $\underset{\mathrm{X}^{a Y Y}}{\mathrm{AB}}$ & $\underset{\mathrm{X}^{a} \mathbf{Y}}{\mathrm{BB}}$ & $\underset{\mathbf{X}^{+} \mathbf{Y}}{\mathrm{AA}}$ & $\underset{\mathbf{X}^{+} \mathbf{Y}}{\mathrm{AB}}$ & $\underset{X+Y}{B B}$ \\
\hline \multirow[t]{2}{*}{$\begin{array}{l}\text { Genotypes } \\
\text { and their } \\
\text { frequencies } \\
\text { in the } \\
\text { males }\end{array}$} & $\begin{array}{l}\mathrm{AAX}^{a} \mathrm{X}^{a} \\
\mathrm{ABX}^{a} \mathrm{X}^{a} \\
\mathrm{BBX}^{a} \mathrm{X}^{a} \\
\mathrm{AAX}^{a} \mathrm{X}^{+} \\
\mathrm{ABX}^{a} \mathrm{X}^{+} \\
\mathrm{BBX} \mathbf{X}^{+} \\
\mathrm{AAX}^{+} \mathrm{X}^{+} \\
\mathrm{ABX}^{+} \mathrm{X}^{+} \\
\mathrm{BBX}^{+} \mathbf{X}^{+}\end{array}$ & $\begin{array}{l}h \\
i \\
j \\
k \\
l \\
m \\
n \\
p \\
q\end{array}$ & $\begin{array}{c}x \\
h x \\
\lambda i x \\
\dddot{k x} \\
\lambda l x \\
\ldots \\
n x \\
\lambda p x \\
\ldots\end{array}$ & $\begin{array}{c}y \\
h y \\
\lambda i y \\
\ldots \\
k y \\
\lambda l y \\
\ldots \\
n y \\
\lambda p y \\
\ldots\end{array}$ & $\begin{array}{c}z \\
h z \\
\lambda i z \\
\ldots \\
k z \\
\lambda l z \\
\ldots \\
n z \\
\lambda p z \\
\ldots\end{array}$ & $\begin{array}{l}r \\
h r \\
i r \\
j r \\
k r \\
l r \\
m r \\
n r \\
p r \\
q r\end{array}$ & $\begin{array}{l}\text { s } \\
h s \\
\text { is } \\
j s \\
k s \\
l s \\
m s \\
n s \\
p s \\
q s\end{array}$ & $\begin{array}{l}t \\
h t \\
i t \\
j t \\
k t \\
l t \\
m t \\
n t \\
p t \\
q t\end{array}$ \\
\hline & Divisors & & $\mathbf{T}$ & $\mathrm{T}$ & $\mathrm{T}$ & $\mathrm{I}$ & I & I \\
\hline
\end{tabular}

$\mathrm{T}=h+k+n+\lambda i+\lambda l+\lambda p . \quad \lambda$ specifies the degree of dominance of the allele A. Dominance is complete when $\lambda=\mathrm{I}$ and recessiveness is complete when $\lambda=0$. 


\section{SEX-LINKED MODELS}

In the sex-linked models there are six different female genotypes and nine different male genotypes. The different possible matings are shown in table I. I am using here the same notation as in my previous paper. The offspring produced by these matings form the population in the next generation. The genotypic frequencies become

$$
\begin{aligned}
& x^{\prime}=\left(x+\frac{1}{2} y\right)\left(h+\frac{1}{2} k+\frac{1}{2} \lambda i+\frac{1}{4} \lambda l\right) / \mathrm{T}+\left(r+\frac{1}{2} s\right)\left(h+\frac{1}{2} i+\frac{1}{2} k+\frac{1}{4} l\right) \\
& y^{\prime}=\left(z+\frac{1}{2} y\right)\left(h+\frac{1}{2} k+\frac{1}{2} \lambda i+\frac{1}{4} \lambda l\right) / \mathrm{T}+\left(x+\frac{1}{2} y\right)\left(\frac{1}{2} \lambda i+\frac{1}{4} \lambda l\right) / \mathrm{T} \\
&+\left(r+\frac{1}{2} s\right)\left(j+\frac{1}{2} i+\frac{1}{2} m+\frac{1}{4} l\right)+\left(t+\frac{1}{2} s\right)\left(h+\frac{1}{2} i+\frac{1}{2} k+\frac{1}{4} l\right) \\
& z^{\prime}=\left(z+\frac{1}{2} y\right)\left(\frac{1}{2} \lambda i+\frac{1}{4} \lambda l\right) / \mathrm{T}+\left(t+\frac{1}{2} s\right)\left(j+\frac{1}{2} i+\frac{1}{2} m+\frac{1}{4} l\right) \\
& r^{\prime}=\left(x+\frac{1}{2} y\right)\left(n+\frac{1}{2} k+\frac{1}{2} \lambda p+\frac{1}{4} \lambda l\right) / \mathrm{T}+\left(r+\frac{1}{2} s\right)\left(n+\frac{1}{2} p+\frac{1}{2} k+\frac{1}{4} l\right) \\
& s^{\prime}=\left(z+\frac{1}{2} y\right)\left(n+\frac{1}{2} k+\frac{1}{2} \lambda p+\frac{1}{4} \lambda l\right) / \mathrm{T}+\left(x+\frac{1}{2} y\right)\left(\frac{1}{2} \lambda p+\frac{1}{4} \lambda l\right) / \mathrm{T} \\
&+\left(r+\frac{1}{2} s\right)\left(q+\frac{1}{2} p+\frac{1}{2} m+\frac{1}{4} l\right)+\left(t+\frac{1}{2} s\right)\left(n+\frac{1}{2} p+\frac{1}{2} k+\frac{1}{4} l\right) \\
& t^{\prime}=\left(z+\frac{1}{2} y\right)\left(\frac{1}{2} \lambda p+\frac{1}{4} \lambda l\right) / \mathrm{T}+\left(t+\frac{1}{2} s\right)\left(q+\frac{1}{2} p+\frac{1}{2} m+\frac{1}{4} l\right) \\
& h^{\prime}=\left(x+\frac{1}{2} y\right)\left(h+\frac{1}{2} k+\frac{1}{2} \lambda i+\frac{1}{4} \lambda l\right) / \mathrm{T} \\
& i^{\prime}=\left(z+\frac{1}{2} y\right)\left(h+\frac{1}{2} k+\frac{1}{2} \lambda i+\frac{1}{4} \lambda l\right) / \mathrm{T}+\left(x+\frac{1}{2} y\right)\left(\frac{1}{2} \lambda i+\frac{1}{4} \lambda l\right) / \mathrm{T} \\
& j^{\prime}=\left(z+\frac{1}{2} y\right)\left(\frac{1}{2} \lambda i+\frac{1}{4} \lambda l\right) / \mathrm{T} \\
& k^{\prime}=\left(x+\frac{1}{2} y\right)\left(n+\frac{1}{2} k+\frac{1}{2} \lambda p+\frac{1}{4} \lambda l\right) / \mathrm{T}+\left(r+\frac{1}{2} s\right)\left(h+\frac{1}{2} i+\frac{1}{2} k+\frac{1}{4} l\right) \\
& l^{\prime}=\left(z+\frac{1}{2} y\right)\left(n+\frac{1}{2} k+\frac{1}{2} \lambda p+\frac{1}{2} \lambda l\right) / \mathrm{T}+\left(x+\frac{1}{2} y\right)\left(\frac{1}{2} \lambda p+\frac{1}{4} \lambda l\right) / \mathrm{T} \\
& \quad+\left(r+\frac{1}{2} s\right)\left(j+\frac{1}{2} i+\frac{1}{2} m+\frac{1}{2} l\right)+\left(t+\frac{1}{2} s\right)\left(h+\frac{1}{2} i+\frac{1}{2} k+\frac{1}{4} l\right) \\
& m^{\prime}=\left(z+\frac{1}{2} y\right)\left(\frac{1}{2} \lambda p+\frac{1}{2} \lambda l\right) / \mathrm{T}+\left(t+\frac{1}{2} s\right)\left(j+\frac{1}{2} i+\frac{1}{2} m+\frac{1}{4} l\right) \\
& n^{\prime}=\left(r+\frac{1}{2} s\right)\left(n+\frac{1}{2} p+\frac{1}{2} k+\frac{1}{4} l\right) \\
& p^{\prime}=\left(r+\frac{1}{2} s\right)\left(q+\frac{1}{2} p+\frac{1}{2} m+\frac{1}{4} l\right)+\left(t+\frac{1}{2} s\right)\left(n+\frac{1}{2} p+\frac{1}{2} k+\frac{1}{4} l\right) \\
& q^{\prime}=\left(t+\frac{1}{2} s\right)\left(q+\frac{1}{2} p+\frac{1}{2} m+\frac{1}{4} l\right) .
\end{aligned}
$$

Let $u, v$ and $w$ be the frequencies of the genotypes $\mathrm{AA}, \mathrm{AB}$ and $\mathrm{BB}$. If $A$ is recessive $(\lambda=0)$ then in my earlier paper it was shown that in the next generation these frequencies become

$$
\begin{aligned}
u^{\prime} & =\left(x+\frac{1}{2} y\right)\left(w+\frac{1}{2} v\right)+\left(u+\frac{1}{2} v\right)^{2} \\
v^{\prime} & =\left(z+\frac{1}{2} y\right)\left(w+\frac{1}{2} v\right)-\left(x+\frac{1}{2} y\right)\left(w+\frac{1}{2} v\right)+2\left(u+\frac{1}{2} v\right)\left(w+\frac{1}{2} v\right) .
\end{aligned}
$$

But if we put $x+y+z=a_{f}$ and $p=u+\frac{1}{2} v$ then

$$
p^{\prime}=\left(l 1-\frac{1}{2} \alpha_{f}\right) p+\frac{1}{2} \alpha_{f} \text {. }
$$

This recurrence relation cannot be applied directly because $a_{f}$ changes from generation to generation. If $\mathrm{A}$ is dominant, however, the recurrence relations are

$$
\begin{gathered}
u^{\prime}=\left(x+\frac{1}{2} y\right)\left(u+\frac{1}{2} v\right) /(u+v)-\left(x+\frac{1}{2} y\right)\left(u+\frac{1}{2} v\right)+\left(u+\frac{1}{2} v\right)^{2} \\
v^{\prime}=\left(z+\frac{1}{2} y\right)\left(u+\frac{1}{2} v\right) /(u+v)+\frac{1}{2} v\left(x+\frac{1}{2} y\right) /(u+v)-\left(x+\frac{1}{2} y\right)\left(w+\frac{1}{2} v\right) \\
-\left(z+\frac{1}{2} y\right)\left(u+\frac{1}{2} v\right)+2\left(u+\frac{1}{2} v\right)\left(w+\frac{1}{2} v\right) .
\end{gathered}
$$

Near equilibrium when terms containing $w, v^{2}$ and higher powers of $v$ can be neglected, the recurrence relations become simply

$$
v^{\prime} \cong v \text {. }
$$

As may be expected selection is much slower if $\mathrm{A}$ is dominant than if $\mathrm{A}$ is recessive. 
Of more interest is the selection of the allele $a$. If $a_{f}$ and $a_{m}$ are the frequencies in females and in males of the chromosome $\mathrm{X}^{\mathrm{a}}$, then

$$
\begin{aligned}
& a_{f}^{\prime}=a_{f}\left(h+\frac{1}{2} k+\lambda i+\frac{1}{2} \lambda l\right) / \mathrm{T}+a_{m}\left(\mathrm{I}-a_{f}\right) \\
& a_{m}{ }^{\prime}=a_{f}\left(h+\frac{3}{4} k+\frac{1}{2} n+\lambda i+\frac{3}{4} \lambda l+\frac{1}{2} \lambda p\right) / \mathrm{T}+\frac{1}{2} a_{m}\left(\mathrm{I}-a_{f}\right) .
\end{aligned}
$$

These equations are true even if $\mathrm{A}$ and $a$ are arbitrarily linked. If linkage is very close we are concerned mostly with the genotypes $\mathrm{X}^{\boldsymbol{a} A} \mathrm{X}^{\mathrm{a} A}, \mathrm{X}^{\mathrm{a} A} \mathrm{X}^{+B}$ and $\mathrm{X}^{+B} \mathrm{X}^{+B}$. If $\mathrm{A}$ is recessive the equations which $I$ gave in my previous paper are

$$
\begin{aligned}
& a_{f^{\prime}} \cong a_{f}+a_{m}\left(\mathrm{I}-a_{f}\right) \\
& a_{m} \cong a_{f}+\frac{1}{2} a_{m}\left(\mathrm{I}-a_{f}\right) .
\end{aligned}
$$

As an example of the rate of selection, if the starting values are $a_{f}=a_{m}=0 \cdot \mathrm{I}$, then after only seven generations $a_{f}=0.997$ and $a_{m}=0.985$. If $\mathrm{A}$ and $a$ are closely linked but $\mathrm{A}$ is dominant then

$$
\begin{aligned}
a_{f^{\prime}} & =a_{f} a_{m} /\left(a_{m}+\frac{1}{2} v_{m}\right)+a_{m}\left(\mathrm{I}-a_{f}\right) \\
a_{m}^{\prime} & =a_{f}\left(a_{m}+1 \frac{1}{4} v_{m}\right) /\left(a_{m}+\frac{1}{2} v_{m}\right)+\frac{1}{2} a_{m}\left(\mathrm{I}-a_{f}\right) \\
v_{m}^{\prime} & =\frac{1}{2} v_{m} a_{f} /\left(a_{m}+\frac{1}{2} v_{m}\right)+a_{m}\left(\mathrm{I}-a_{f}\right),
\end{aligned}
$$

where $v_{m}$ is the frequency of the genotype $\mathrm{X}^{a A} \mathrm{X}^{+B}$. At equilibrium $v_{m}=0$ and $a_{f}=a_{m}=\mathrm{I}$. The population therefore consists entirely of the genotypes $\mathrm{X}^{a A} \mathrm{Y}$ and $\mathrm{X}^{a A} \mathrm{X}^{\mathrm{a} A}$.

At the start of selection we can safely assume that $a_{f} a_{m}$ is negligible. If $A$ is recessive this leads to the general equations $I$ gave in my earlier paper.

$$
\begin{aligned}
& \left(a_{f}\right)_{n} \cong(\mathrm{I} \cdot 7808)^{n}\left\{(\mathrm{I} \cdot 2808)\left(a_{f}\right)_{0}+\left(a_{m}\right)_{0}\right\} /(2 \cdot 06 \mathrm{I} 6), \\
& \left(a_{m}\right)_{n} \cong(\mathrm{I} \cdot 7808)^{n}\left\{(0 \cdot 7808)\left(a_{m}\right)_{0}+\left(a_{f}\right)_{0}\right\} /(2 \cdot 06 \mathrm{I} 6) .
\end{aligned}
$$

\begin{tabular}{|c|c|c|}
\hline \multirow{2}{*}{ Generation } & \multicolumn{2}{|c|}{ Gene frequency of $A$ in females } \\
\hline & A dominant & A recessive \\
\hline $\begin{array}{l}0 \\
1 \\
2 \\
3 \\
4 \\
5 \\
6 \\
7\end{array}$ & $\begin{array}{l}0 \cdot 00001 \\
0 \cdot 000015 \\
0 \cdot 0000219 \\
0 \cdot 0000331 \\
0 \cdot 0000500 \\
0 \cdot 0000759 \\
0 \cdot 000115 \\
0 \cdot 000175\end{array}$ & $\begin{array}{l}0 \cdot 00001 \\
0 \cdot 00002 \\
0 \cdot 000035 \\
0 \cdot 0000625 \\
0 \cdot 000111 \\
0 \cdot 000198 \\
0 \cdot 000353 \\
0 \cdot 000628\end{array}$ \\
\hline
\end{tabular}

Unfortunately the divisor $2 \cdot 06 \mathrm{I} 6$ was omitted in proof and I failed to correct the error. It is the determinant of the matrix of characteristic vectors. If $\mathbf{A}$ is dominant, however, a general solution for the initial rate of selection cannot be found. But we can easily compare the rates of selection for particular starting values. For example if at the start $a_{f}=a_{m}=0.0000 \mathrm{I}$, then in successive generations we have 
When the alleles $\mathrm{A}$ and $a$ are either unlinked or not so closely linked that crossing-over between them can be ignored, we must return to the equations

$$
\begin{aligned}
& a_{f^{\prime}}=a_{f}\left(h+\frac{1}{2} k+\lambda i+\frac{1}{2} \lambda l\right) / \mathrm{T}+a_{m}\left(\mathrm{I}-a_{f}\right) \\
& a_{m}{ }^{\prime}=a_{f}\left(h+\frac{3}{4} k+\frac{1}{2} n+\lambda i+\frac{3}{4} \lambda l+\frac{1}{2} \lambda p\right) / \mathrm{T}+\frac{1}{2} a_{m}\left(\mathrm{I}-a_{f}\right) .
\end{aligned}
$$

At the start of selection we can again assume that $a_{f}$ and $a_{m}$ are so small that quadratic and higher terms in $a$ can be neglected. This means that $h$ and $i$ and terms like $a_{f} k$ and $a_{f} l$ are negligible. Thus if A is dominant

Therefore

$$
\begin{aligned}
& a_{f^{\prime}} \cong a_{m} \\
& a_{m} \cong \frac{1}{2} a_{f}+\frac{1}{2} a_{m} .
\end{aligned}
$$

$$
a_{m}{ }^{\prime \prime} \cong \frac{1}{2} a_{m}{ }^{\prime}+\frac{1}{2} a_{m}
$$

This equation has the general solution

$$
\left(a_{m}\right)_{n}=\mathrm{P}+\mathrm{Q}\left(-\frac{1}{2}\right)^{n}
$$

where $\mathrm{P}+\mathrm{Q}=\left(a_{m}\right)_{0}$ and $\mathrm{P}-\frac{1}{2} \mathrm{Q}=\left(a_{m}\right)_{1}$. After only a few generations the value of $a$ would settle down to $\frac{1}{3}\left(a_{m}\right)_{0}+\frac{2}{3}\left(a_{m}\right)_{1}$. The mating preference would not increase in the population. Even in the absence of linkage, however, an association must develop between the advantageous genotypes and the mating preference genes. Thus $h, i, k$ and $l$ become relatively more important. Selection, although slow at first, later speeds up. The same equation holds if $\mathrm{A}$ is recessive (O'Donald, 1962).

Near equilibrium we can assume that $h+k+n+i+l+p=\mathrm{I}$. If $\mathrm{A}$ is dominant, then again

$$
\begin{aligned}
& a_{f}^{\prime} \cong a_{m} \\
& a_{m}{ }^{\prime} \cong \frac{1}{2} a_{f}+\frac{1}{2} a_{m} .
\end{aligned}
$$

As expected the rate of selection falls off. If $A$ is recessive, however, a simple approximation cannot be found. But we can put $h+k+n$ $\cong l_{\mathrm{I}}-v$ where $v$ is the frequency of the heterozygotes $\mathrm{AB}$. When the frequency of the $a$ gene is high the heterozygotes are rapidly eliminated from the population in accordance with the equation $v^{\prime}=\left(1-\frac{1}{2} a_{f}\right) v$. After a long period of selection it can intuitively be seen that $h+\frac{1}{2} k \cong a_{m}$, for a close association must arise between the preferred genotypes and the mating preference genes. Calculation shows this to be so. Further if $v$ is small, $i$ and $l$ are very small and

$$
a_{f} a_{m}=a_{f}\left(h+\frac{1}{2} k+i+\frac{1}{2} l\right) \cong a_{f}\left(h+\frac{1}{2} k\right) \text {. }
$$

When all terms in $v^{2}$ and higher powers of $v$ are neglected we have

$$
\begin{aligned}
& a_{f}^{\prime} \cong a_{m}+a_{f} a_{m} v \\
& a_{m}{ }^{\prime} \cong \frac{1}{2} a_{f}(\mathrm{I}+v)+\frac{1}{2} a_{m}+\frac{1}{2} a_{f} a_{m} v .
\end{aligned}
$$

Even if $a_{f}$ and $a_{m}$ remain rather small, they still increase because

$$
a_{m}{ }^{\prime \prime} \cong \frac{1}{2} a_{m}{ }^{\prime}+\frac{1}{2} a_{m}\left(\mathrm{I}+v^{\prime}\right) .
$$


Linkage, however, will ensure that $a_{f} a_{m} v$ is not a negligible factor. Thus the selection of a linked recessive will always be fast. There seems no doubt not only that selection will pick out linked combinations of genes but also that recessive male genes will be even more rapidly picked out.

\section{AUTOSOMAL MODELS}

If $\mathrm{A}$ and $a$ are both autosomal genes there are nine genotypes each having the same frequency in both females and males. The frequencies of the different possible matings in the selection of a dominant are shown in table 2. I assume throughout that the mating

TABLE 2

\begin{tabular}{|c|c|c|c|c|c|c|c|c|c|c|}
\hline & & \multicolumn{9}{|c|}{ Genotypes and their frequencies in the females } \\
\hline & & $\begin{array}{l}\mathrm{AA} \\
a a\end{array}$ & $\begin{array}{l}\mathrm{AA} \\
a+\end{array}$ & $\begin{array}{l}\mathbf{A A} \\
++\end{array}$ & $\begin{array}{c}\mathrm{AB} \\
a a\end{array}$ & $\begin{array}{l}\mathrm{AB} \\
a+\end{array}$ & $\begin{array}{l}\mathbf{A B} \\
++\end{array}$ & $\begin{array}{l}\text { BB } \\
a a\end{array}$ & $\begin{array}{c}\text { BB } \\
a+\end{array}$ & $\begin{array}{l}\mathrm{BB} \\
++\end{array}$ \\
\hline \multirow{10}{*}{$\begin{array}{l}\text { Frequencies of } \\
\text { corresponding } \\
\text { genotypes } \\
\text { in the males }\end{array}$} & & $r$ & $s$ & $t$ & $u$ & $v$ & $w$ & $x$ & $y$ & $z$ \\
\hline & $r$ & $r^{2}$ & rs & $r t$ & $r u$ & $r v$ & $r w$ & $r x$ & $r y$ & $r z$ \\
\hline & $s$ & rs & $s^{2}$ & $s t$ & $s u$ & $s v$ & $s w$ & $s x$ & sy & $s z$ \\
\hline & $t$ & $r t$ & $s t$ & $t^{2}$ & $t u$ & tv & $t w$ & $t x$ & ty & $t z$ \\
\hline & $u$ & $r u$ & $s u$ & tu & $u^{2}$ & $u v$ & $u w$ & $u x$ & $u y$ & $u z$ \\
\hline & $v$ & $r v$ & $s v$ & tv & $u v$ & $v^{2}$ & $v w$ & $v x$ & $v y$ & $v z$ \\
\hline & $w$ & $r w$ & $s w$ & $t w$ & $u w$ & $v w$ & $w^{2}$ & $w x$ & $w y$ & $w z$ \\
\hline & $x$ & $\ldots$ & $\cdots$ & $t x$ & $\cdots$ & $\ldots$ & $w x$ & $\cdots$ & $\cdots$ & $x z$ \\
\hline & $y$ & $\cdots$ & $\cdots$ & $t y$ & $\cdots$ & $\cdots$ & $w y$ & $\cdots$ & $\cdots$ & $\begin{array}{l}y z \\
-8\end{array}$ \\
\hline & $z$ & $\cdots$ & $\cdots$ & & $\cdots$ & $\cdots$ & $w z$ & $\cdots$ & $\cdots$ & $z^{\circ}$ \\
\hline \multicolumn{2}{|c|}{ Divisors } & $\mathbf{T}$ & $\mathbf{T}$ & I & $\mathrm{T}$ & $\mathrm{T}$ & I & $\mathbf{T}$ & $\mathrm{T}$ & I \\
\hline
\end{tabular}

$\mathrm{T}=r+s+t+u+v+w$.

preference acts as a dominant. This is certainly the most realistic assumption if sexual selection is to be effective when $a$ is low in frequency. From table 2 we can obtain recurrence relations which equate the frequencies of the genotypes $a a$ and $a+$ from one generation to the next. If these frequencies are $u_{a}$ and $v_{a}$ respectively then it can be shown that

$$
\begin{aligned}
& u_{a}^{\prime}=\left(u_{a}+\frac{1}{2} v_{a}\right)\left(r+\frac{1}{2} s+u+\frac{1}{2} v\right) /(r+s+t+u+v+w) \\
& v_{a}^{\prime}=\frac{1}{2} v_{a}+u_{a}\left(t+\frac{1}{2} s+w+\frac{1}{2} v\right) /(r+s+t+u+v+w)+w_{a}\left(u_{a}+\frac{1}{2} v_{a}\right) .
\end{aligned}
$$

Thus unlike the selection of a sex-linked preference we find recurrence relations in terms of the genotypic frequencies rather than the gene frequency. If $\mathrm{A}$ is recessive the corresponding recurrence relations are

$$
\begin{aligned}
& u_{a}{ }^{\prime}=\left(u_{a}+\frac{1}{2} v_{a}\right)\left(r+\frac{1}{2} s\right) /(r+s+t) \\
& v_{a}^{\prime}=\frac{1}{2} v_{a}+u_{a}\left(t+\frac{1}{2} s\right) /(r+s+t)+w_{a}\left(u_{a}+\frac{1}{2} v_{a}\right) .
\end{aligned}
$$


I consider first the effect of close linkage. When $\mathrm{A}$ is dominant

$$
\begin{aligned}
& u_{a}^{\prime}=\left(u_{a}+\frac{1}{2} v_{a}\right)^{2} /\left(u_{a}+v_{a}\right) \\
& v_{a}{ }^{\prime}=\frac{1}{2} v_{a}+\frac{1}{2} u_{a} v_{a} /\left(u_{a}+v_{a}\right)+w_{a}\left(u_{a}+\frac{1}{2} v_{a}\right) .
\end{aligned}
$$

At first quadratic terms in $u_{a}$ and $v_{a}$ are negligible. Putting $u_{a}+\frac{1}{2} v_{a}=p_{a}$ we get the equation $p_{a}{ }^{\prime} \cong \frac{3}{2} p_{a}$, or after $n$ generations $\left(p_{a}\right)_{n} \cong(1 \cdot 5)^{n}\left(p_{a}\right)_{0}$. In the final approach to equilibrium $w_{a} \cong 0$, $v_{a}{ }^{\prime} \cong v_{a}$ and selection becomes very slow. If $\mathrm{A}$ is recessive, however, we get the equations

$$
\begin{aligned}
& u_{a}{ }^{\prime}=u_{a}+\frac{1}{2} v_{a} \\
& v_{a}{ }^{\prime}=\frac{1}{2} v_{a}+w_{a}\left(u_{a}+\frac{1}{2} v_{a}\right) .
\end{aligned}
$$

At the start of selection, therefore,

$$
\left(\begin{array}{l}
u_{a}^{\prime} \\
v_{a}^{\prime}
\end{array}\right) \cong\left(\begin{array}{ll}
\mathrm{I} & \frac{1}{2} \\
\mathrm{I} & \mathrm{I}
\end{array}\right)\left(\begin{array}{l}
u_{a} \\
v_{a}
\end{array}\right) .
$$

The dominant latent root of the matrix is $I+I / \sqrt{ } 2$ or $I \cdot 707 \mathrm{I}$. The starting values must be $\left(u_{a}\right)_{0} \cong 0$ and $\left(v_{a}\right)_{10} \cong 2\left(p_{a}\right)_{0}$. After $n$ generations the frequency of the allele $a$ has become

$$
\left(p_{a}\right)_{n} \cong(\mathrm{I} \cdot 707 \mathrm{I})^{n}(\mathrm{I} \cdot 207 \mathrm{I})\left(p_{a}\right)_{0} .
$$

Near equilibrium $v_{a}^{\prime} \cong \frac{1}{2} v_{a}$, a very fast elimination of the heterozygotes. Thus a recessive is always selected much faster than a dominant.

When there is no linkage between $\mathrm{A}$ and $a$ and $\mathrm{A}$ is dominant we must return to the original equations. Clearly when $u_{a} \cong 0 v_{a}^{\prime} \cong v_{a}$ and as expected selection is slow. Again near equilibrium when $r+s+t+u+v+w \cong \mathrm{I}$ we have

$$
\begin{aligned}
& u_{a}^{\prime} \cong\left(u_{a}+\frac{1}{2} v_{a}\right)^{2} \\
& v_{a}^{\prime} \cong \frac{1}{2} v_{a}+u_{a}\left(w_{a}+\frac{1}{2} v_{a}\right)+w_{a}\left(u_{a}+\frac{1}{2} v_{a}\right) .
\end{aligned}
$$

Put $u_{a}+\frac{1}{2} v_{a}=p_{a}$. This gives the equations

$$
\begin{aligned}
& p_{a}{ }^{\prime} \cong p_{a}^{2}+1 v_{a}+\frac{1}{2} u_{a}\left(1-p_{a}\right)+\frac{1}{2} w_{a} p_{a} \\
& \quad \cong p_{a} .
\end{aligned}
$$

The frequency of the $a$ gene does not increase near equilibrium.

If $\mathrm{A}$ is recessive, then from the recurrence relations $v_{a}^{\prime} \cong v_{a}$ at the start and selection is slow. Near equilibrium $r+s+t \cong \mathrm{I}-\mathrm{V}_{A}$, where $V_{A}$ is the frequency of $A B$ genotypes.

$$
\begin{aligned}
& u_{a}^{\prime} \cong\left(u_{a}+\frac{1}{2} v_{a}\right)^{2} /\left(l_{\mathrm{I}}-\mathrm{V}_{A}\right) \\
& v_{a}^{\prime} \cong \frac{1}{2} v_{a}+u_{a}\left(w_{a}+\frac{1}{2} v_{a}\right) /\left(\mathrm{I}-\mathrm{V}_{A}\right)+w_{a}\left(u_{a}+\frac{1}{2} v_{a}\right) .
\end{aligned}
$$

The gene $a$ continues to increase until $\mathrm{V}_{A}=0$.

\section{CONCLUSIONS}

Both sex-linked and autosomal models give roughly the same results. Selection is more rapid if $A$ is recessive than if $A$ is dominant. 
The effect of dominance on the rate of sexual selection is therefore quite the opposite of its effect on the rate of natural selection which is very slow for a recessive gene. I have suggested that one effect of sexual selection will be to pick out those advantageous genes for male display that are closely linked to the female preference genes. Those male genes that are recessive will also be picked out. Modifiers too may be selected. Under sexual selection, therefore, the wild-type genes may become recessive. The genetics of some of the spectacular plumage characters in male birds may thus help to confirm or deny the importance of sexual selection.

These rather unexpected results can be understood without mathematics. Sexual selection depends on an association between the mating preference genes and the preferred male genotypes. Linkage will of course make the association between them closer. At the same time if recessives alone are preferred, the preference genes will not be dispersed among disadvantageous genotypes. Preference for a dominant, however, must disperse the preference genes among disadvantageous alleles when segregation takes place in the heterozygotes.

\section{SUMMARY}

I. Sexual selection depends on the evolution of a female mating preference. Two models are set up-for a mating preference that is either sex-linked or autosomally linked. Within each model the preferred male character may be either dominant or recessive and closely or loosely linked to the mating preference gene.

2. If the preferred male gene and the mating preference gene are close together on the X-chromosome, selection is very fast, especially so if the male gene is recessive. Selection is slower if the male character and the mating preference are unlinked. Even so a recessive will increase more rapidly than a dominant. The autosomal model gives similar results.

3. If sexual selection has been in action, some of the genes that determine the male character may thus have become recessive and have formed a closely linked supergene with the female preference.

\section{REFERENCES}

FISHER, R. A. 1930. The Genetical Theory of Natural Selection. Clarendon Press, Oxford.

O'DONALd, P. 1962. The theory of sexual selection. Heredity, $17,541-55^{2}$. 